\title{
Character Association and Path Coefficient Analysis for Yield and Its Components in Pigeonpea (Cajanus cajan (L.) Mill sp.)
}

\author{
Parsagoni Mallesh ${ }^{1,2 *}$, Nidhi Mohan ${ }^{2,3}$, Jake Lee ${ }^{2,4}$, H.C. Nanda ${ }^{1}$ and \\ C.V. Sameer Kumar ${ }^{2}$ \\ ${ }^{1}$ Department of Genetics and Plant Breeding, Indira Gandhi Agricultural University, \\ Raipur-492012, Chhattisgarh, India \\ ${ }^{2}$ International Crops Research Institute for the Semi-Arid Tropics (ICRISAT), \\ Patancheru-502324, India \\ ${ }^{3}$ Department of Genetics and Plant Breeding, Acharya NG Ranga Agricultural University, \\ Bapatla, Guntur-522 509, India \\ ${ }^{4}$ Department of Horticulture, Benguet state university, Philippines 2601 \\ *Corresponding author
}

\section{A B S T R A C T}

\begin{tabular}{|l|}
\hline Ke y w or d s \\
$\begin{array}{l}\text { Correlation coefficient, } \\
\text { Path analysis, Pollen } \\
\text { fertility and pigeonpea }\end{array}$ \\
\hline Article Info \\
\hline Accepted: \\
22 October 2018 \\
Available Online: \\
10 November 2018 \\
\hline \hline
\end{tabular}

The estimates of genotypic correlation coefficients in general were higher than their corresponding phenotypic correlation coefficients indicating strong inherent association among the traits. The seed yield per plant had significant and positive association with number of primary branches plant ${ }^{-1}$, number of secondary branches plant ${ }^{-1}$, number of pods plant $^{-1}$, number of seeds plant ${ }^{-1}$, biological yield plant ${ }^{-1}$, seed yield $(\mathrm{kg} / \mathrm{ha})$ harvest index and 100 -seed weight at both genotypic as well as phenotypic levels. Characters viz., pollen fertility $\%$, biological yield plant ${ }^{-1}$, harvest index, number of secondary branches plant ${ }^{-1}$ and dal recovery\% exerted high direct effects on seed yield. 100-seed weight and pods plant ${ }^{-1}$ also contributed indirectly towards seed yield per plant through most of the characters. Thus, based on the present study number of primary branches plant ${ }^{-1}$, number of secondary branches plant ${ }^{-1}$, number of pods plant ${ }^{-1}$, number of seeds plant ${ }^{-1}$, biological yield plant ${ }^{-1}$, seed yield $(\mathrm{kg} / \mathrm{ha}$ ) harvest index and 100 -seed weight were emerged as the most important components of seed yield.

\section{Introduction}

Pigeonpea [Cajanus cajan (L.) Mill sp.] is the most important pulse crop of our country, though its productivity is very low. Yield is a complex and multiplicative trait, which is highly influenced by environment. The information on correlation coefficient of yield and yield attributes will assist the breeders whether to give due importance on each of the traits to make significant genetic gain. Path coefficient analysis aids partitioning of the correlation coefficient into direct and indirect effects and thus provides a better insight on the characters and their relationship with yield. The correlation and path analysis thus help in identifying suitable traits for improving the seed yield. The present 
investigation was under taken to assess the association of yield and yield attributes and to estimate the direct and indirect effects of each character on seed yield in pigeonpea.

\section{Materials and Methods}

A total of 28 genotypes including 3 varietal checks (Asha, Maruti, Rajeevlochan) and 2 hybrid checks (ICPH 2740 and ICPH 2671) were grown in randomized complete block design (RCBD) with three replications and two rows per plot each of 4 meter length with a spacing of 75 and $50 \mathrm{~cm}$ between and within rows respectively at International Crops Research Institute for the Semi-Arid Tropics (ICRISAT), Patancheru. The recommended agronomic practices were followed for raising a good and healthy crop. In each replication five plants were selected randomly in each genotype for recording observations on days to $50 \%$ flowering, pollen fertility $\%$, days to maturity, plant height $(\mathrm{cm})$, number of primary branches plant ${ }^{-1}$, number of secondary branches plant ${ }^{-1}$, number of pods plant $^{-1}$, number of seeds $\operatorname{pod}^{-1}$, number of seeds plant $^{-1}, 100$-seed weight $(\mathrm{g})$, seed yield plant $^{-1}$ (g), biological yield plant ${ }^{-1}$, seed yield $(\mathrm{kg}$ $\mathrm{ha}^{-1}$ ), harvest index (\%) and seed protein content were recorded. The data were statistically analyzed to estimate the phenotypic correlation coefficients of yield, yield attributes, Path coefficient analysis was done for yield, and yield attributes to estimate the direct and indirect effects of various characters on seed yield by using the software INDOSTAT.

\section{Results and Discussion}

The analysis of variance indicated significant differences among genotypes for all the characters. The correlation coefficients between seed yield and yield attributes are presented in Table 1. Genotypic correlations (Table 1) were higher in magnitude than the phenotypic correlation indicting strong inherent relationship among the characters except few, which could be due to modifying effects in the environment studied. Pandey and Singh (2002) reported similar findings. Genotypic correlation provides a measure of genotypic association among different traits and helps in identifying the traits in selection. In the present study, seed yield per plant recorded significant and positive association with seed yield plant ${ }^{-1}$ was observed to be significantly and positively associated with number of primary branches plant ${ }^{-1}$, number of secondary branches plant ${ }^{-1}$, number of pods plant $^{-1}$, number of seeds plant ${ }^{-1}$, biological yield plant $^{-1}$ and seed yield $(\mathrm{kg} / \mathrm{ha})$ at both phenotypic and genotypic levels and harvest index showed positive association both phenotypic and genotypic but significant only genotypic level. However, seed yield plant $^{-1}$ was also observed to be significantly and negatively associated with days to $50 \%$ flowering, days to maturity and pollen fertility\%. Association of seed yield plant ${ }^{-1}$ with other characters, viz., plant height, number of seeds pod $^{-1}, 100$-seed weight and at both phenotypic and genotypic levels was found but non-significant. However, it recorded negative and significant association with plant height, days to $50 \%$ flowering, days to maturity and straw yield at phenotypic level (Table 1). The results of the present study are in conformity with the reports of Aher et al., (1998); Musaana and Nahdy (1998) and Chandirakala and Subbaraman (2010). Among the yield contributing traits, positive and significant association were evident for days to maturity with plant height, days to $50 \%$ flowering with days from flowering to maturity; seeds per pod with number of primary branches and pod length; days to50\% flowering with plant height as well as specific gravity with test weight; which are in conformity with earlier reports (Salunke et al., 1995, Vikas and Singh, 1998, Baskaran and Muthiah, 2006, Sodavadiya et al., 2009, 
Sawant et al., 2009). However, VasanthaRao et al., (2010) reported non-significant but positive association of test weight with seed yield.

Studies on inter-character associations among the yield components studied had revealed significant and positive association of days to $50 \%$ flowering with days to maturity, pollen fertility $\%$ and 100 -seed weight; days to maturity with pollen fertility and seed yield $(\mathrm{kg} / \mathrm{ha})$; number of primary branches plant ${ }^{-1}$ with number of secondary branches plant ${ }^{-1}$, plant height, biological yield plant ${ }^{-1}$ and seed yield $(\mathrm{kg} / \mathrm{ha})$.

Number of secondary branches per plant with number of pods plant ${ }^{-1}$; number of seeds plant ${ }^{-}$ ${ }^{1}$, biological yield plant $^{-1}$ and seed yield ( $\mathrm{kg} / \mathrm{ha})$; plant height with 100 -seed weight; number of pods plant ${ }^{-1}$ with number of seeds plant $^{-1}$, biological yield plant ${ }^{-1}$ and seed yield $(\mathrm{kg} / \mathrm{ha})$; Number of seeds plant $^{-1}$ with biological yield plant ${ }^{-1}$ and seed yield $(\mathrm{kg} / \mathrm{ha})$; biological yield plant ${ }^{-1}$ with seed yield $(\mathrm{kg} / \mathrm{ha})$ similarly, seed yield (kg/ha) with harvest index positive and significant at both the phenotypic and genotypic level indicating the possibility of simultaneous improvement of these characters through selection.

However significant and negative inter character association was observed for days to $50 \%$ flowering with number of primary branches plant ${ }^{-1}$, number of pods plant ${ }^{-1}$, number of seeds plant ${ }^{-1}$, biological yield plant $^{-1}$ and seed yield (kg/ha); days to maturity with number of primary branches plant ${ }^{-1}$, number of pods plant ${ }^{-1}$ and biological yield plant $^{-1}$; biological yield plant $^{-1}$ with harvest index at phenotypic and genotypic levels, indicating competition for a common possibility, such as nutrient supply (Adams, 1967; Adams and Grafius, 1971) and the need for balanced selection, while attempting for improvement of these traits.
The characters viz., number of primary branches plant $^{-1}$, number of secondary branches plant ${ }^{-1}$, number of pods plant ${ }^{-1}$, number of seeds plant ${ }^{-1}$, biological yield plant ${ }^{1}$, seed yield $(\mathrm{kg} / \mathrm{ha})$ harvest index and 100seed weight recorded positive association with seed yield, which indicated that these are major yield contributing traits in pigeonpea. Hence, laying stress on these traits will aid in making significant improvement of yield potentiality in pigeonpea.

Path coefficient analysis considers direct as well as indirect effects of the variables by partitioning the correlation coefficients. The detailed path coefficient analysis (Table 2) showed that pollen fertility $\%$ had maximum positive direct effect fallowed by biological yield plant $^{-1}$, harvest index, number of secondary branches plant ${ }^{-1}$ and dal recovery $\%$. Whereas, the characters viz., days to $50 \%$ flowering, days to maturity, and seed protein content showed negative and high direct effects on grain yield at both genotypic and phenotypic levels. Whereas, number of seeds plant $^{-1}$, number of primary branches, 100 -seed weight and number of seeds $\operatorname{pod}^{-1}$ in these cases genotypic had showed negative direct effect but phenotypic had showed positive and direct effects on the seed yield plant ${ }^{-1}$.

Plant height and number of pods plant ${ }^{-1}$ at genotypic level had showed positive direct effect but negative direct effect on the grain yield at phenotypic level. The highly significant and positive correlation of pollen fertility $\%$ had found with grain yield due to their maximum direct and indirect effect via days to $50 \%$ flowering and days to maturity respectively. Number of seeds pod $^{-1}$ showed medium positive direct effect and their genotypic correlation with grain yield was significant. Similar results were also reported by Rao et al., (2013) for number of primary branches, pods plant ${ }^{-1}$, seeds pod $^{-1}$, days to $50 \%$ flowering and harvest index. 
Table.1 Phenotypic and Genotypic correlations for yield and yield components in pigeonpea genotypes

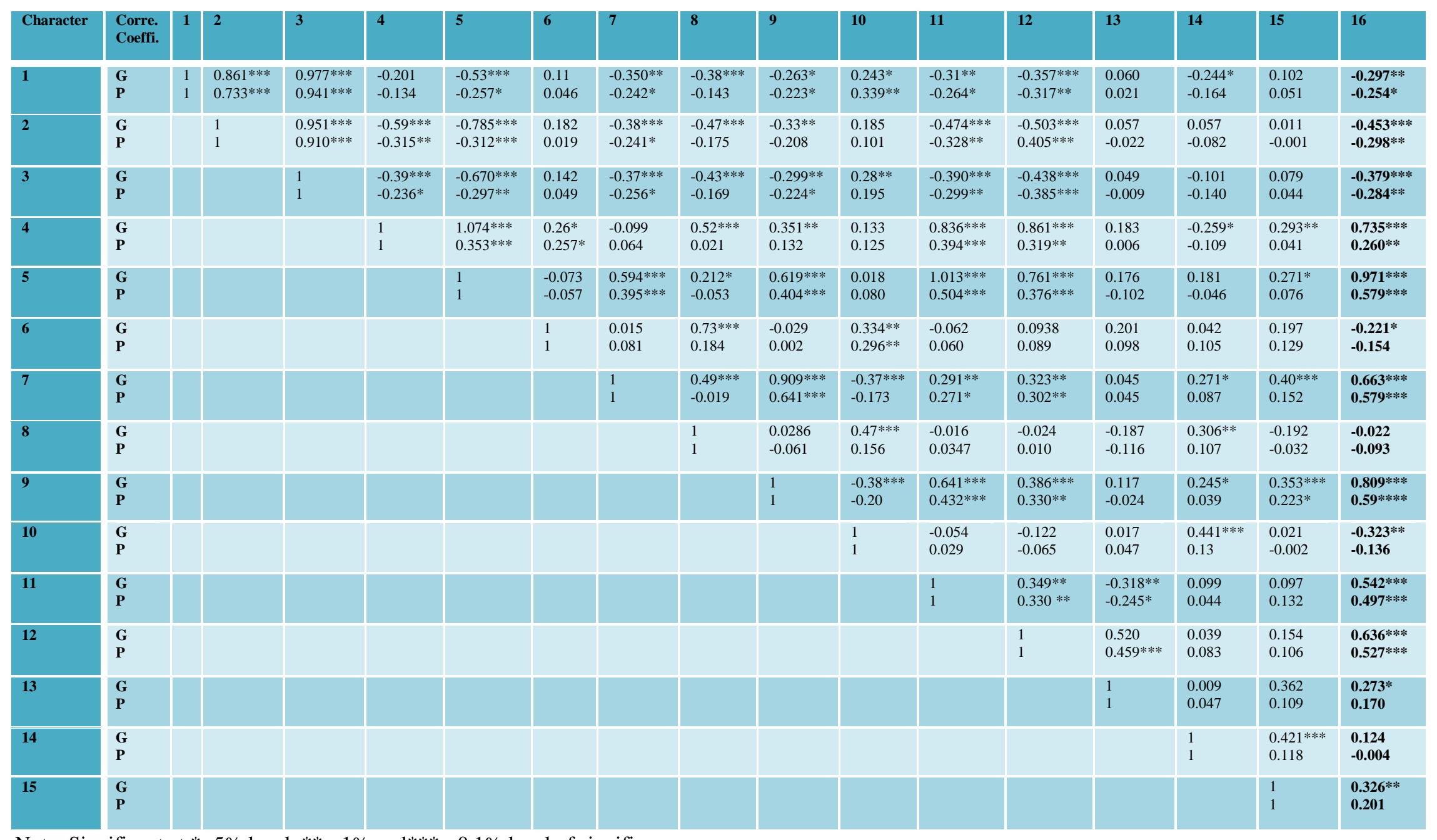

Note: Significant at $*-5 \%$ level, $* *-1 \%$ and $* * *-0.1 \%$ level of significance

Note: 1 - Days to $50 \%$ flowering, 2- Days to maturity, 3- Pollen fertility, 4- No. of primary branches plant ${ }^{-1}, 5$-No.of secondary branches plant ${ }^{-1}, 6$ - Plant height, 7 Pods plant $^{-1}, 8$-Seeds pod $^{-1}, 9$-Seeds plant ${ }^{-1}, 10$ - 100-seed weight, 11-Biological yield plant ${ }^{-1}, 12$ - Yield (kg/ha), 13- Harvest index, 14- seed protein content, 15 Dal recovery\% and 16-Yield plant $^{-1}$. 
Table.2 Genotypic and Phenotypic path co-efficient for yield and yield components in pigeonpea

\begin{tabular}{|c|c|c|c|c|c|c|c|c|c|c|c|c|c|c|c|c|}
\hline Character & Pathcoeff. & 1 & 2 & 3 & 4 & 5 & 6 & 7 & 8 & 9 & 10 & 11 & 12 & 13 & 14 & $r_{g}$ and $r_{p}$ \\
\hline 1 & $\begin{array}{l}\mathbf{G} \\
\mathbf{P}\end{array}$ & $\begin{array}{l}-13.13 \\
-0.362 \\
\end{array}$ & $\begin{array}{l}-10.121 \\
-0.388\end{array}$ & $\begin{array}{l}23.331 \\
-0.499\end{array}$ & $\begin{array}{l}0.182 \\
0.071\end{array}$ & $\begin{array}{l}-0.068 \\
0.136\end{array}$ & $\begin{array}{l}0.023 \\
-0.024\end{array}$ & $\begin{array}{l}-0.353 \\
0.128\end{array}$ & $\begin{array}{l}0.072 \\
0.076\end{array}$ & $\begin{array}{l}0.325 \\
0.118\end{array}$ & $\begin{array}{l}-0.083 \\
-0.129\end{array}$ & $\begin{array}{l}-0.577 \\
0.139\end{array}$ & $\begin{array}{l}0.071 \\
-0.011\end{array}$ & $\begin{array}{l}0.000 \\
-0.027\end{array}$ & $\begin{array}{l}0.034 \\
0.087\end{array}$ & $\begin{array}{l}-0.297 * * \\
-0.254 *\end{array}$ \\
\hline 2 & $\begin{array}{l}\mathbf{G} \\
\mathbf{P}\end{array}$ & $\begin{array}{l}-11.309 \\
-0.275\end{array}$ & $\begin{array}{l}-11.752 \\
\underline{-0.223}\end{array}$ & $\begin{array}{l}22.814 \\
-0.341\end{array}$ & $\begin{array}{l}0.609 \\
0.118\end{array}$ & $\begin{array}{l}-0.099 \\
0.117\end{array}$ & $\begin{array}{l}0.038 \\
-0.007\end{array}$ & $\begin{array}{l}-0.384 \\
0.090\end{array}$ & $\begin{array}{l}0.089 \\
0.066\end{array}$ & $\begin{array}{l}0.408 \\
0.078\end{array}$ & $\begin{array}{l}-0.045 \\
-0.038\end{array}$ & $\begin{array}{l}-0.883 \\
0.123\end{array}$ & $\begin{array}{l}0.068 \\
0.008\end{array}$ & $\begin{array}{l}0.000 \\
0.001\end{array}$ & $\begin{array}{l}-0.008 \\
0.031\end{array}$ & $\begin{array}{l}-0.453 * * * * \\
-0.298 * *\end{array}$ \\
\hline 3 & $\begin{array}{l}\mathbf{G} \\
\mathbf{P}\end{array}$ & $\begin{array}{l}-12.804 \\
0.837\end{array}$ & $\begin{array}{l}-11.206 \\
0.809\end{array}$ & $\begin{array}{l}23.942 \\
\underline{0.547}\end{array}$ & $\begin{array}{l}0.381 \\
-0.210\end{array}$ & $\begin{array}{l}-0.085 \\
-0.265\end{array}$ & $\begin{array}{l}0.030 \\
0.043\end{array}$ & $\begin{array}{l}-0.379 \\
-0.228\end{array}$ & $\begin{array}{l}0.080 \\
-0.151\end{array}$ & $\begin{array}{l}0.377 \\
-0.199\end{array}$ & $\begin{array}{l}-0.069 \\
0.173\end{array}$ & $\begin{array}{l}-0.728 \\
-0.266\end{array}$ & $\begin{array}{l}0.066 \\
-0.008\end{array}$ & $\begin{array}{l}0.000 \\
0.039\end{array}$ & $\begin{array}{l}0.015 \\
-0.125\end{array}$ & $\begin{array}{l}-0.379 * * * \\
-0.284 * *\end{array}$ \\
\hline 4 & $\begin{array}{l}\mathbf{G} \\
\mathbf{P}\end{array}$ & $\begin{array}{l}2.380 \\
-0.012\end{array}$ & $\begin{array}{l}7.132 \\
-0.029\end{array}$ & $\begin{array}{l}-9.083 \\
-0.022\end{array}$ & $\frac{-1.014}{\underline{0.122}}$ & $\begin{array}{l}0.138 \\
0.033\end{array}$ & $\begin{array}{l}0.049 \\
0.024\end{array}$ & $\begin{array}{l}-0.064 \\
0.006\end{array}$ & $\begin{array}{l}-0.102 \\
0.002\end{array}$ & $\begin{array}{l}-0.452 \\
0.012\end{array}$ & $\begin{array}{l}-0.032 \\
0.012\end{array}$ & $\begin{array}{l}1.565 \\
0.037\end{array}$ & $\begin{array}{l}0.215 \\
0.001\end{array}$ & $\begin{array}{l}0.001 \\
0.004\end{array}$ & $\begin{array}{l}0.044 \\
-0.010\end{array}$ & $\begin{array}{l}0.735 * * * \\
0.260 * *\end{array}$ \\
\hline 5 & $\begin{array}{l}\mathbf{G} \\
\mathbf{P}\end{array}$ & $\begin{array}{l}7.072 \\
-0.056\end{array}$ & $\begin{array}{l}9.333 \\
-0.067\end{array}$ & $\begin{array}{l}-16.277 \\
-0.064\end{array}$ & $\begin{array}{l}-1.108 \\
0.076\end{array}$ & $\underline{\underline{0.117}}$ & $\begin{array}{l}-0.017 \\
-0.013\end{array}$ & $\begin{array}{l}0.599 \\
0.085\end{array}$ & $\begin{array}{l}-0.042 \\
-0.011\end{array}$ & $\begin{array}{l}-0.774 \\
0.087\end{array}$ & $\begin{array}{l}-0.005 \\
0.017\end{array}$ & $\begin{array}{l}1.894 \\
0.108\end{array}$ & $\begin{array}{l}0.209 \\
-0.022\end{array}$ & $\begin{array}{l}0.001 \\
0.016\end{array}$ & $\begin{array}{l}-0.026 \\
-0.010\end{array}$ & $\begin{array}{l}0.971 * * * * \\
0.579 * * *\end{array}$ \\
\hline 6 & $\begin{array}{l}\mathbf{G} \\
\mathbf{P}\end{array}$ & $\begin{array}{l}-1.433 \\
-0.011\end{array}$ & $\begin{array}{l}-2.126 \\
-0.005\end{array}$ & $\begin{array}{l}3.419 \\
-0.012\end{array}$ & $\begin{array}{l}-0.234 \\
-0.063\end{array}$ & $\begin{array}{l}-0.01 \\
0.014\end{array}$ & $\begin{array}{l}\frac{0.212}{-0.270} \\
\underline{0}\end{array}$ & $\begin{array}{l}0.014 \\
-0.020\end{array}$ & $\begin{array}{l}-0.138 \\
-0.046\end{array}$ & $\begin{array}{l}0.037 \\
-0.000\end{array}$ & $\begin{array}{l}-0.081 \\
-0.074\end{array}$ & $\begin{array}{l}-0.115 \\
-0.015\end{array}$ & $\begin{array}{l}0.239 \\
-0.024\end{array}$ & $\begin{array}{l}0.001 \\
-0.032\end{array}$ & $\begin{array}{l}-0.006 \\
-0.026\end{array}$ & $\begin{array}{l}-0.221 * \\
-0.154\end{array}$ \\
\hline 7 & $\begin{array}{l}\mathbf{G} \\
\mathbf{P}\end{array}$ & $\begin{array}{l}4.593 \\
-0.073\end{array}$ & $\begin{array}{l}4.472 \\
-0.072\end{array}$ & $\begin{array}{l}-8.984 \\
-0.077\end{array}$ & $\begin{array}{l}0.064 \\
0.019\end{array}$ & $\begin{array}{l}0.074 \\
0.118\end{array}$ & $\begin{array}{l}0.003 \\
0.024\end{array}$ & $\begin{array}{l}\frac{1.008}{-0.390} \\
\end{array}$ & $\begin{array}{l}-0.095 \\
-0.006\end{array}$ & $\begin{array}{l}-1.122 \\
0.192\end{array}$ & $\begin{array}{l}0.090 \\
-0.051\end{array}$ & $\begin{array}{l}0.542 \\
0.081\end{array}$ & $\begin{array}{l}0.054 \\
0.014\end{array}$ & $\begin{array}{l}0.002 \\
0.046\end{array}$ & $\begin{array}{l}-0.038 \\
0.026\end{array}$ & $\begin{array}{l}0.663 * * * \\
0.579 * * *\end{array}$ \\
\hline 8 & $\begin{array}{l}\mathbf{G} \\
\mathbf{P}\end{array}$ & $\begin{array}{l}4.951 \\
-0.001\end{array}$ & $\begin{array}{l}5.524 \\
-0.001\end{array}$ & $\begin{array}{l}-10.008 \\
-0.001\end{array}$ & $\begin{array}{l}-0.537 \\
0.000\end{array}$ & $\begin{array}{l}0.027 \\
-0.000\end{array}$ & $\begin{array}{l}0.154 \\
0.001\end{array}$ & $\begin{array}{l}0.505 \\
-0.000\end{array}$ & $\frac{-0.191}{\underline{0.007}}$ & $\begin{array}{l}-0.035 \\
-0.001\end{array}$ & $\begin{array}{l}-0.114 \\
0.001\end{array}$ & $\begin{array}{l}-0.031 \\
0.000\end{array}$ & $\begin{array}{l}-0.222 \\
-0.001\end{array}$ & $\begin{array}{l}-0.001 \\
-0.000\end{array}$ & $\begin{array}{l}-0.043 \\
0.001\end{array}$ & $\begin{array}{l}-0.022 \\
-0.093\end{array}$ \\
\hline 9 & $\begin{array}{l}\mathbf{G} \\
\mathbf{P}\end{array}$ & $\begin{array}{l}3.457 \\
-0.032\end{array}$ & $\begin{array}{l}3.885 \\
-0.03\end{array}$ & $\begin{array}{l}-7.306 \\
-0.033\end{array}$ & $\begin{array}{l}-0.368 \\
0.019\end{array}$ & $\begin{array}{l}0.079 \\
0.059\end{array}$ & $\begin{array}{l}-0.006 \\
0.000\end{array}$ & $\begin{array}{l}0.918 \\
0.093\end{array}$ & $\begin{array}{l}-0.006 \\
-0.009\end{array}$ & $\frac{-1.238}{\underline{0.195}}$ & $\begin{array}{l}0.092 \\
-0.029\end{array}$ & $\begin{array}{l}1.193 \\
0.063\end{array}$ & $\begin{array}{l}0.139 \\
-0.004\end{array}$ & $\begin{array}{l}0.001 \\
0.033\end{array}$ & $\begin{array}{l}-0.034 \\
0.006\end{array}$ & $\begin{array}{l}0.809 * * * * \\
0.59 * * * *\end{array}$ \\
\hline 10 & $\begin{array}{l}\mathbf{G} \\
\mathbf{P}\end{array}$ & $\begin{array}{l}-4.448 \\
-0.005\end{array}$ & $\begin{array}{l}-2.179 \\
-0.002\end{array}$ & $\begin{array}{l}6.74 \\
-0.004\end{array}$ & $\begin{array}{l}-0.134 \\
-0.003\end{array}$ & $\begin{array}{l}0.002 \\
-0.002\end{array}$ & $\begin{array}{l}0.071 \\
-0.006\end{array}$ & $\begin{array}{l}-0.374 \\
0.004\end{array}$ & $\begin{array}{l}-0.089 \\
-0.003\end{array}$ & $\begin{array}{l}0.474 \\
0.004\end{array}$ & $\frac{-0.242}{0.288}$ & $\begin{array}{l}-0.101 \\
-0.001\end{array}$ & $\begin{array}{l}0.019 \\
-0.001\end{array}$ & $\begin{array}{l}0.000 \\
0.000\end{array}$ & $\begin{array}{l}-0.062 \\
-0.003\end{array}$ & $\begin{array}{l}-0.323 * * \\
-0.136\end{array}$ \\
\hline 11 & $\begin{array}{l}\mathbf{G} \\
\mathbf{P}\end{array}$ & $\begin{array}{l}4.074 \\
-0.057\end{array}$ & $\begin{array}{l}5.574 \\
-0.071\end{array}$ & $\begin{array}{l}-9.362 \\
-0.065\end{array}$ & $\begin{array}{l}-0.845 \\
0.085\end{array}$ & $\begin{array}{l}0.129 \\
0.109\end{array}$ & $\begin{array}{l}-0.013 \\
0.013\end{array}$ & $\begin{array}{l}0.294 \\
0.059\end{array}$ & $\begin{array}{l}0.003 \\
0.008\end{array}$ & $\begin{array}{l}-0.791 \\
0.094\end{array}$ & $\begin{array}{l}0.013 \\
0.006\end{array}$ & $\begin{array}{l}\underline{1.858} \\
\underline{0.289}\end{array}$ & $\begin{array}{l}-0.377 \\
-0.053\end{array}$ & $\begin{array}{l}0.000 \\
0.029\end{array}$ & $\begin{array}{l}-0.015 \\
0.009\end{array}$ & $\begin{array}{l}0.542 * * * * \\
0.497 * * *\end{array}$ \\
\hline 12 & $\begin{array}{l}\mathbf{G} \\
\mathbf{P}\end{array}$ & $\begin{array}{l}-0.791 \\
0.004\end{array}$ & $\begin{array}{l}-0.673 \\
-0.004\end{array}$ & $\begin{array}{l}1.334 \\
-0.001\end{array}$ & $\begin{array}{l}-0.182 \\
0.001\end{array}$ & $\begin{array}{l}0.022 \\
-0.019\end{array}$ & $\begin{array}{l}0.043 \\
0.019\end{array}$ & $\begin{array}{l}0.046 \\
0.009\end{array}$ & $\begin{array}{l}0.036 \\
-0.023\end{array}$ & $\begin{array}{l}-0.145 \\
-0.005\end{array}$ & $\begin{array}{l}-0.004 \\
0.009\end{array}$ & $\begin{array}{l}-0.592 \\
-0.048\end{array}$ & $\underline{1.184}$ & $\begin{array}{l}0.001 \\
0.021\end{array}$ & $\begin{array}{l}-0.001 \\
0.01\end{array}$ & $\begin{array}{l}0.273^{*} \\
0.170\end{array}$ \\
\hline 13 & $\begin{array}{l}\mathbf{G} \\
\mathbf{P}\end{array}$ & $\begin{array}{l}-1.342 \\
0.003\end{array}$ & $\begin{array}{l}-0.125 \\
0.0001\end{array}$ & $\begin{array}{l}1.471 \\
0.003\end{array}$ & $\begin{array}{l}-0.308 \\
0.002\end{array}$ & $\begin{array}{l}0.035 \\
0.004\end{array}$ & $\begin{array}{l}0.042 \\
0.008\end{array}$ & $\begin{array}{l}0.406 \\
0.009\end{array}$ & $\begin{array}{l}0.037 \\
-0.000\end{array}$ & $\begin{array}{l}-0.436 \\
0.013\end{array}$ & $\begin{array}{l}-0.005 \\
-0.000\end{array}$ & $\begin{array}{l}0.181 \\
0.001\end{array}$ & $\begin{array}{l}0.428 \\
0.006\end{array}$ & $\begin{array}{l}\underline{0.004} \\
\underline{0.059}\end{array}$ & $\begin{array}{l}-0.059 \\
0.007\end{array}$ & $\begin{array}{l}0.326 * * \\
0.201\end{array}$ \\
\hline 14 & $\begin{array}{l}\mathbf{G} \\
\mathbf{P}\end{array}$ & $\begin{array}{l}3.205 \\
0.003\end{array}$ & $\begin{array}{l}-0.669 \\
0.002\end{array}$ & $\begin{array}{l}-2.623 \\
0.003\end{array}$ & $\begin{array}{l}0.318 \\
0.002\end{array}$ & $\begin{array}{l}0.023 \\
0.000\end{array}$ & $\begin{array}{l}0.009 \\
-0.002\end{array}$ & $\begin{array}{l}0.273 \\
-0.001\end{array}$ & $\begin{array}{l}-0.058 \\
-0.002\end{array}$ & $\begin{array}{l}-0.303 \\
-0.001\end{array}$ & $\begin{array}{l}-0.107 \\
-0.003\end{array}$ & $\begin{array}{l}0.186 \\
-0.001\end{array}$ & $\begin{array}{l}0.011 \\
0.001\end{array}$ & $\begin{array}{l}0.002 \\
0.001\end{array}$ & $\begin{array}{l}-\frac{-0.141}{-0.019} \\
\underline{\underline{-0}}\end{array}$ & $\begin{array}{l}0.124 \\
-0.004\end{array}$ \\
\hline
\end{tabular}

Residual effect $=0.4788$ for Genotypic path and Residual effect $=0.5680$ for Phenotypic path coefficients. Diagonal values $=$ direct effects Off-diagonal values $=$ indirect effects.

Note: 1-Days to $50 \%$ flowering, 2-Days to maturity, 3-Pollen fertility\%, 4-No.of primary branches plant ${ }^{-1}, 5$-No.of secondary branches plant ${ }^{-1}$, 6-Plant height, 7-

Pods plant ${ }^{-1}, 8$-Seeds pod ${ }^{-1}$,9-Seeds plant ${ }^{-1}, 10-100$-seed weight, 11-Biological yield plant ${ }^{-1}, 12$-Harvest index, 13-Dal recovery\% and 14-seed protein content.

$r_{\mathrm{g}}$ and $\mathrm{r}_{\mathrm{p}}-$ Genotypic correlation and Phenotypic correlation 
Considering overall observation of path analysis the traits viz., pollen fertility\%, biological yield plant $^{-1}$, harvest index, pods plant $^{-1}$ and number of primary branches plant ${ }^{-1}$ showed considerable positive direct influence on seed yield plant ${ }^{-1}$.

However, the character pollen fertility\% did not exhibit positive correlation on yield due to its high negative indirect effect via days to $50 \%$ flowering, days to maturity, pods plant ${ }^{-1}$ and biological yield plant ${ }^{-1}$. Hence, improving seed yield of pigeonpea may be possible through selection for these traits.

From the present investigations here concluded that the estimation of correlation and path analysis are more useful to identification of superior cultivars for yield and yield traits. Based on association studies, improvement in pigeonpea can be attained by isolating individuals possessing high values for the characters like primary branchesplant 1 , number of secondary branches plant ${ }^{-1}$, number of pods plant ${ }^{-1}$, number of seeds plant $^{-1}$ and biological yield plant ${ }^{-1}$.

The character seed yield plant ${ }^{-1}$ had high to moderate positive indirect effects via characters viz., pods plant ${ }^{-1}$, seeds plant ${ }^{-1}$, number of secondary branches plant ${ }^{-1}$ and biological yield plant $^{-1}$ also exhibited the significant and positive association with seed yield.

These traits may be given more emphasis while selecting high yielding pigeon pea genotypes for drought condition.

\section{Acknowledgements}

The first author wish to acknowledge International crops research institute for the semi-arid tropics (ICRISAT), Patancheru for providing financial support for research through internship.

\section{References}

Adams, M.W. and Grafius, J.E.1971. Yieldcomponents compensation alternative interpretations. Crop Sci., 11: 33-35.

Aher, R. P., Thombre, B. B. and Dahal, D. V., 1998, Genetic variability and character association in pigeonpea [Cajanus cajan (L.) Mill sp.]. Legume Res., 21(1): 4144.

Baskaran, K. and Muthiah, A. R. 2006, Variability studies in pigeonpea [Cajanus cajan (L.) Mill sp.]. Res. on Crops, 7(1): 249-252.

Chandirakala, R. and Subbaraman, N., 2010, Character association andpath analysis for yield attributes in full sib progenies in pigeonpea [Cajanus cajan (L.) Mill sp.]. Electronic J. Pl. Breed., 1(4): 824827.

Dewey, D. R. and Lu, K. N., 1959, A correlation and path coefficient analysis of components of crested wheat grass seed production. Agron. J., 51: 515-518.

Musaana, M. S. and Nahdy, M. S., 1998, Path coefficient analysis of yield and its components in pigeonpea. African Crop Sci. J., 6: 143-148.

Pandey, N. and Singh, N. B. 2002.Association between yield and yield attribute in pigeonpea Madras Agric. J. 88: 640643.

Rao, P. J. M, Malathi, S, Reddy, D.V.V, Upender, M. 2013. Genetic Studies of Association and Path Coefficient Analysis of Yield and its Component Traits in Pigeon Pea (Cajanus cajan (L.) Mill sp.). International Journal of Scientific and Research Publications, 3(8): $1-5$.

Sodavadiya, P. R., Pithia, M. S., Savaliya, J. J., Pansuriya, A. G. and Korat, V. P., 2009, Studies on characters association and path analysis for seed yield and its components in pigeonpea [Cajanus 
cajan (L.) Mill sp.]. Legume Res., 32 (3): 203-205.

Thanki, H. P. and Sawargaonkar, S. L., 2010, Path coefficient analysis in pigeonpea (Cajanus cajanL. Millsp.). Electronic J. Pl.Breed., 1(4): 936-939

Vasantha Rao, U., Govinda Rao, B., Panduranga Rao, C and Srinivasa Rao, V., 2010. Character association and path coefficient analyses for yield and component traits in pigeonpea [Cajanus cajan (L.) Mill sp.]. The Andhra Agril. J., 57(3): 298-300.
Vikas and Singh, S. P., 1998, Variability and character association in early and extra early genotypes of pigeonpea [Cajanus cajan (L.) Mill sp.]. Legume Res., 21(3/4): 229-232.

Weber C. R. and Murthy, B. R., 1952, Heritable and non-heritable relationship and variability of oil content and agronomic characteristics in the F2 generation of soybean crosses. Agron.J., 44: 202-209.

\section{How to cite this article:}

Parsagoni Mallesh, Nidhi Mohan, Jake Lee, H.C. Nanda and Sameer Kumar, C.V. 2018. Character Association and Path Coefficient Analysis for Yield and its Components in Pigeonpea (Cajanus cajan (L.) Mill sp.). Int.J.Curr.Microbiol.App.Sci. 7(11): 2770-2776. doi: https://doi.org/10.20546/ijcmas.2018.711.318 